\title{
Localización de empresas: de la teoría a la práctica apegada al terreno
}

\section{Enterprise localisation; from theory to practice in the territorial context}

GarRIDO, RubÉn (2007), LoCALIZACIÓN Y MOVILIDAD DE EMPRESAS en España, Fundación eol-Unión Europea-Fondo Social EuropeoM inisterio de Industria, Turism O y COM ERCIO, AlCalá de H ENARES, 132 PP., ISBN : 978-84-88723-74-1.

El libro de Rubén Garrido, profesor de economía de la Universidad de Alcalá de $\mathrm{H}$ enares, marca un importante parteaguas en el análisis de los fenómenos de localización y deslocalización de las actividades económicas en España. Además, el texto es valioso para comprender y explicar los determinantes del entorno geoeconómico y la variedad de factores que determinan la localización y movilidad de empresas en el entorno regional de las economías de mercado.

De acuerdo con lo señalado por el autor, el motivo central que da vida a este estudio se relaciona con el efecto de la globalización económica en la dinámica de concentración-difusión de las actividades económicas, lo que tiene implicaciones en términos de la distribución espacial de la actividad económica en los ámbitos mundial y local.

Cabe destacar que la unidad de análisis que utiliza el autor es la empresa ubicada en el territorio español. En efecto, la preocupación central en el trabajo se relaciona con los factores que determinan las actividades y las formas de control de las empresas, como los cambios tecnológicos que han facilitado desarroIlar fases de producción estandarizables con costos bajos, los cuales permiten a las empresas tener mucho mayores posibilidades de movilidad internacional.

Por ello, la economía internacional se caracteriza por una tendencia a la globalización de las actividades económicas liderada por las empresas, en particular multinacionales, lo que sin duda determina una mayor importancia de las regiones, donde se presenta una dura competencia por atraer capital e inversiones de empresas que permitan promover el crecimiento económico en los ámbitos nacional e internacional. 
Así pues, uno de los grandes aciertos del libro es el tratamiento de los aspectos teóricos de la localización de las empresas de una manera pragmática 0 , como el autor manifiesta, "más pegada al terreno" (6), en el contexto de las tendencias predominantes hacia la globalización y la integración de las actividades económicas del sistema económico mundial actual. En esa perspectiva, en el segundo capítulo del texto se presenta una síntesis del escenario global caracterizado por el desarrollo de tecnologías, la intensificación del comercio, de los flujos financieros, la consolidación de las empresas multinacionales como motores del crecimiento basado en la producción flexible y descentralizada, y en la creciente importancia de las instituciones multilaterales, que contradictoriamente coexisten con la consolidación de acuerdos comerciales preferenciales entre diferentes regiones del mundo, liderada por la integración de la Unión Europea y seguida del surgimiento de acuerdos como el TLCAN, el M ercosur y la APEC.

Es en este contexto de dinámica económica globalizada, en el que las regiones compiten por adoptar procesos productivos derivados de la deslocalización con base en los costos, la infraestructura, el capital humano y el capital social disponible localmente. Con justa razón el autor subraya el surgimiento de la competencia interterritorial y la importancia de los contextos locales.

O tro aspecto que se debe destacar es el que se relaciona con el recuento de los aspectos más importantes de la literatura sobre teoría de la localización. La revisión parte de la escuela alemana donde sobresalen los trabajos de Von Thünen, Weber, Chistaller y Lösch. Los aspectos fundamentales que se distinguen de este desarrollo teórico son, por una parte, la consideración del espacio como fuente de ingresos y costos (asociados al transporte) y, por otra, la visión de la teoría de los lugares centrales que definen al espacio en términos de la proximidad de mercados, como el eje de la demanda de los consumidores.

La revisión incluye el fenómeno de las aglomeraciones industriales y urbanas que permiten abordar el desarrollo de los procesos de industrialización y la creciente urbanización que los acompaña. Esta perspectiva analítica se fundamenta en el concepto de economía externa, cuya conceptualización se deriva de los planteamientos incluidos en el libro Principios de economía, de Alfred $\mathrm{M}$ arshall, sobre el desarrollo de distritos industriales, con base en la existencia de mercados de trabajo especializados, la disponibilidad de insumos especializados y el desbordamiento o difusión tecnológica en una región determinada. El resultado 
de este ambiente de actividades fuera de la operación interna de la empresa, permite reducir costos más allá de las condiciones técnicas de operación. Es importante destacar, como menciona el autor, que a partir del trabajo seminal de $M$ arshall se han desarrollado diferentes vertientes de análisis fundamentadas en la idea de externalidades.

De esta manera, se mencionan los trabajos de Jacobs que subrayan cómo la diversidad tecnológica se desarrolla en el contexto de una estructura de mercado competitiva y de la diversidad industrial en una región urbana. Además, por su parte K rugman, Fujita y Venables analizan el contexto de competencia monopólica con el enfoque de nueva geografía económica para explicar la realidad de las regiones, resaltando que tanto la transferencia de tecnología como el mercado laboral especializado se han considerado de manera menos rigurosa en los modelos empíricos, ya que se asumen de manera implícita dentro de estos (por medio de economías de escala y relaciones intraindustriales). Por ello, los autores establecen un modelo para captar los costos de transporte y los flujos monetarios. Además, dentro de la perspectiva de la localización se destacan, también, los trabajos de Arrow y Romer que indican que la especialización industrial, en un contexto de concentración de mercado, puede generar economías externas por la difusión tecnológica al interior de una misma industria.

Considerando la naturaleza y objetivos del texto, la revisión teórica del concepto de localización es más que suficiente, ya que incluso incorpora las principales críticas a este enfoque por parte de las teorías behavioristas e institucionalistas. Así pues, la revisión en su conjunto permite al lector tener una visión general del fenómeno de la localización desde el punto de vista de teoría económica regional.

Vale la pena mencionar la importancia del concepto de aglomeraciones y su relación con las economías externas en la literatura sobre el análisis económico regional. De acuerdo con M orrison y Siegel, las externalidades generadas por la aglomeración económica han sido relevantes para explicar los temas de productividad, los ciclos económicos, el crecimiento económico regional y la teoría de la localización, entre otros. En este sentido, algunos autores resaltan que las economías de aglomeración son relevantes para explicar los aspectos económicos de la formación y crecimiento de las ciudades modernas, como es el caso de N akamura, Krugman y Livas, y H enderson, Kuncoro y Turner, entre otros. En esta 
perspectiva, $\mathrm{H}$ enderson y Krugman han subrayado que la existencia de fuerzas centrípetas y centrífugas determinan las tendencias del crecimiento urbano manufacturero.

En todo caso se debe destacar el espléndido trabajo de asimilación y síntesis de las principales aproximaciones (neoclásica, behaviorista e institucional) a la perspectiva microeconómica relacionada con las decisiones de localización de las empresas en el espacio. Las distinciones de los enfoques se realizan con base en el estado de competencia del mercado, la natural eza del espacio y las relaciones económicas, la toma de decisiones y los cambios de la localización en el largo plazo.

O tro aspecto que identifica al libro se refiere al esfuerzo realizado por el autor para utilizar específicamente los principales postulados de la teoría de la localización para establecer una tipología de temas que permiten identificar las características tangibles e intangibles que determinan la localización de empresas en una región. En este sentido, el autor identifica y explica las siguientes condiciones: el transporte, los insumos y el tamaño del mercado; el factor trabajo, las economías externas urbanas y de localización; las infraestructuras económicas, sociales y culturales; las condiciones del suelo, el capital físico y financiero, el medio ambiente y el gobierno.

Con base en estos aspectos y adaptando un trabajo de $\mathrm{H}$ ayter, se desarrolla un análisis de las características tangibles e intangibles que determinan las condiciones de localización. Sin duda, esta tarea de definición de características intangibles en el contexto del objetivo de estudio de localización de empresas del libro, es importante pues permite tocar piso en aspectos como la seguridad, disponibilidad y riesgo del transporte, los sindicatos, la rotación del factor laboral, la existencia de externalidades positivas, la disponibilidad de infraestructura y de suelo, las actitudes sobre el medio ambiente, así como la estabilidad y clima empresarial generado por las acciones del gobierno.

Con el fin de adaptar los aspectos teóricos al análisis concreto de la realidad de las empresas que operan en España, y considerando que la localización de empresas se basa en decisiones complejas que incorporan diferentes aspectos, Garrido destaca y desarrolla particularidades del proceso de internalización de la empresa dando el salto del en-torno al in-torno. En esta perspectiva, el autor señala que las empresas enfrentan diferentes variables y dan distinta importancia (localizaciones condicionadas, óptimas y satisfactorias) a estas variables en la toma de decisiones de loca- 
lización. Finalmente, en este apartado se indica que los factores específicos para cada empresa se relacionan al menos con el tipo de empresa (multinacional o Pyme), el tipo de establecimiento, el ciclo de vida y las características de los productos, además de los factores como energía y mano de obra especializada.

Los apartados 3, 4 y 5 del libro abordan el análisis empírico de la movilidad empresarial en España. Este estudio se basa en una encuesta realizada en el año 2005 a 250, sobre todo, pequeñas y medianas empresas localizadas en ese país, en particular en las comunidades de M adrid, Cataluña, Valencia y Andalucía. La descripción de la muestra destaca las características de las empresas, su distribución regional, su división por tipo de actividad y origen de capital, la intensidad y la trayectoria tecnológica.

Los resultados de la encuesta destacan que en España las empresas tienen una mayor vinculación con proveedores del entorno nacional y regional que con el exterior, no obstante, alrededor de la mitad son exportadoras. Un aspecto fundamental que se deriva de la aplicación de la encuesta se relaciona con el grado de complejidad que tienen las decisiones de localización de las empresas derivadas de los aspectos más valorados positivamente por las firmas, como el acceso a clientes, proveedores e información, la mano de obra calificada, las infraestructuras tecnológicas y equipamientos urbanos; así como los aspectos valorados de forma negativa, como los costos de mano de obra, el precio del suelo, la proximidad a empresas del mismo sector y los incentivos financieros y fiscales. Aún más, el llamado ajuste locacional se deriva también de factores como los cambios de mercado, de preferencias de consumidores, regulaciones ambientales, progreso tecnológico, entre otros.

Un tema que se menciona en relación con los factores tangibles e intangibles tiene que ver con las ventajas competitivas de las empresas españolas, las cuales enfrentan la competencia de los nuevos socios de la Comunidad Europea, caracterizados porque tienen economías que ofrecen menores costos de factores de producción, como el precio del suelo y la mano de obra. Dos últimos aspectos que enriquecen el texto se relacionan con la búsqueda de indicadores que permitan identificar las fuerzas (de expulsión y atracción) que mueven la localización de empresas. Para ello el autor se vale de dos técnicas: la elaboración de un índice de complejidad y el establecimiento de un modelo logit multinomial para estimar probabilidades en las decisiones de localización. 
En primer término, el índice relaciona el conjunto de factores incluidos en la encuesta con las empresas con base en la clasificación de importancia. Cuanto mayores factores existan en las decisiones de localización más elevado será el valor de dicho índice. Con base en este indicador, el autor puntualiza que, en general, las empresas se moverían por aspectos relacionados con los procesos internos de la firma, como el precio del suelo. Sin embargo, al dividirlas por su nivel de intensidad tecnológica la necesidad de ampliación sería el factor más importante.

En la parte final del texto se presenta un modelo econométrico logit multinomial que relaciona la probabilidad de localizarse en las diferentes regiones de España con un vector de variables de características generales de las empresas, otro que aglutina factores que dan ventaja a la localización y uno más con variables relacionadas con ventajas competitivas y la competencia internacional. Los resultados muestran que las empresas de tecnología media y alta prefieren ubicarse en la región de Cataluña. Las de intensidad media-baja presentan un panorama más diversificado prefiriendo M adrid, Cataluña y la Comunidad Valenciana.

Para concluir, cabe destacar las diferencias de este estudio microeconómico en el ámbito de regiones en España con lo que ha acontecido en la ubicación de empresas manufactureras de M éxico. Por ejemplo, la apertura e integración a la economía de Estados Unidos ha traído consecuencias negativas en el nivel de especialización y la generación de economías de escala, y en general en el efecto de las economías externas generadas por el cambio de localización de empresas que han favorecido la frontera norte del país. En efecto, la apertura comercial constituyó un elemento clave en la decisión de localización, sobre todo para aquella industria fuertemente vinculada con el sector externo, como es el caso del sector manufacturero. Sin embargo, la razón por la cual la industria no se localiza totalmente en la frontera norte se debe a que la región central mantiene un liderazgo en términos de mercado interno. En este sentido, queda claro que el cambio en la tendencia del crecimiento del empleo manufacturero reciente muestra un proceso de dispersión de la industria manufacturera, favoreciendo a la frontera norte y los estados alrededor de la ciudad de M éxico. Sin embargo, las consecuencias de esto se reflejan en una dimensión menor de las aglomeraciones actuales, aunado a menos especialización de las regiones, la ausencia de economías de escala y un debilitamiento en los encadenamientos del sector manufacturero. 
Finalmente, la lectura del libro, sin lugar a dudas, deja temas importantes para ser retomados por la academia en ciencias sociales, a fin de desarrollar un análisis microeconómico más aterrizado de los problemas económicos y sociales del día y además es un estímulo para que se realicen esfuerzos de sincronización entre la problemática a estudiar, en el que la teoría explicativa se relacione estrechamente con el diseño de la encuesta en términos de las variables que se identifican y que enriquecen las visiones teóricas. Así pues, el libro es ampliamente recomendable para la comunidad de investigadores no sólo de la disciplina económica, sino para el conjunto de las ciencias sociales.

Recibida: 16 de enero de 2008. Aceptada: 28 de enero de 2008.

Jorge Eduardo M endoza-Cota Colegio de la Frontera N orte Correo-e: emendoza@colef.mx

Jorge Echardo Mendoza. Es doctor por la Universidad de Utah, es investigador del Departamento de Estudios Económicos de El Colegio de la Frontera N orte, donde fue director entre 2003 y 2007. También fue coordinador de las maestrías en economía regional de la Universidad Autónoma de Coahuila y en economía aplicada de El Colegio de la Frontera N orte. Es investigador nivel ॥ del SNI. Imparte clases de economía regional, crecimiento económico y economía laboral en maestría y doctorado, tanto en El Colegio de la Frontera N orte como en la Universidad de San Diego. Entre sus múltiples publicaciones destacan: “EI TLCAN y la integración económica de la frontera M éxico-Estados Unidos: situación presente y estrategias a futuro", Foro Internacional, xLV (181), El Colegio de M éxico, M éxico, pp. 517-544 (2005); “Specialization, Agglomeration and Urban $M$ anufacturing Growth in the N orthern Border Cities of M exico", Journal of Borderlands Studies, 16 (2), Association of Borderland Scholars, Texas A\& M International University, pp. 71-98 (2001), "Un modelo de externalidades para el crecimiento manufacturero regional", Estudios Económicos, 14 (2), El Colegio de M éxico, M éxico, pp. 231264 (2000). 